\title{
Desmoplastic ganglioglioma
}

\author{
Report of a non-infantile case
}

\author{
Flávio Ramalho Romero', Sérgio Listik', Paula Annunciato Fabris
}

Desmoplastic infantile gangliogliomas (DIGs) are rare, superficial, supratentorial tumours of early childhood i.e. they occur within the first two years of life representing $1.25 \%$ of all intracranial tumors in children ${ }^{1,2}$. Tumours with similar characteristics are exceedingly rare in the non infantile population ${ }^{1}$. These tumours, which are composed of a mixture of glial and neuronal cells and a fibrous stroma, affect mainly young patients and arise at the surface of the cerebral hemispheres ${ }^{1,2}$. Despite its histologically malignant appearance, DIGs are associated with excellent prognoses ${ }^{1-3}$.

A 19 year old young man presented with history of a year old headache and generalized tonic clonic seizures 3 days before consultation. MRI showed a right temporal lobe solid-cystic lesion, causing mass effect and cerebral edema (Fig 1A). The patient was operated by craniotomy and micro neurosurgery and the lesion totally removed. Light microscopy revealed a cellular glioneuronal tumour showing lobules of dysplastic neuronal cells and multinucleated giant cells. Immunohisto- chemistry demonstrated GFAP positive glial component and synaptophysin and chromogranin positive ganglion cells.

Post operative MRI showed no evidence of residual lesion (Fig 1B). The patient and his parents agree with this report.

In 1982 Taratuto et al. $^{2}$ defined desmoplastic infantile astrocytomas as meningocerebral astrocytomas attached to the dura mater with a desmoplastic reaction. Five years later VandenBerg et al., ${ }^{2,3}$ recognized a ganglion cell component in part of these tumours, and such tumours were called "desmoplastic infantile gangliogliomas".

Desmoplastic infantile gangliogliomas are a distinct form of developmental neuroepithelial tumours probably arising from neural progenitor cells in the subcortical zone along with mature subpial astrocytes ${ }^{3}$. They are rare WHO Grade I tumours of infancy characterized by large volume, superficial location, invariable supratentoriality, fronto-parietal lobe predilection and morphologically by an admixture of astroglial and neuroepithelial elements in

\section{Correspondence}

Flávio Ramalho Romero Rua Pascoal Vita 366 / apto 94 05445-000 São Paulo SP - Brasil E-mail: frromero@ig.com.br romeroncr@gmail.com

Received 4 October 2010

Received in final form 13 0ctober 2010 Accepted 20 October 2010
Fig 1. [A] MRI showing a right temporal solidcystic lesion in Axial view. [B] Pos operative $M R I$ in axial view.
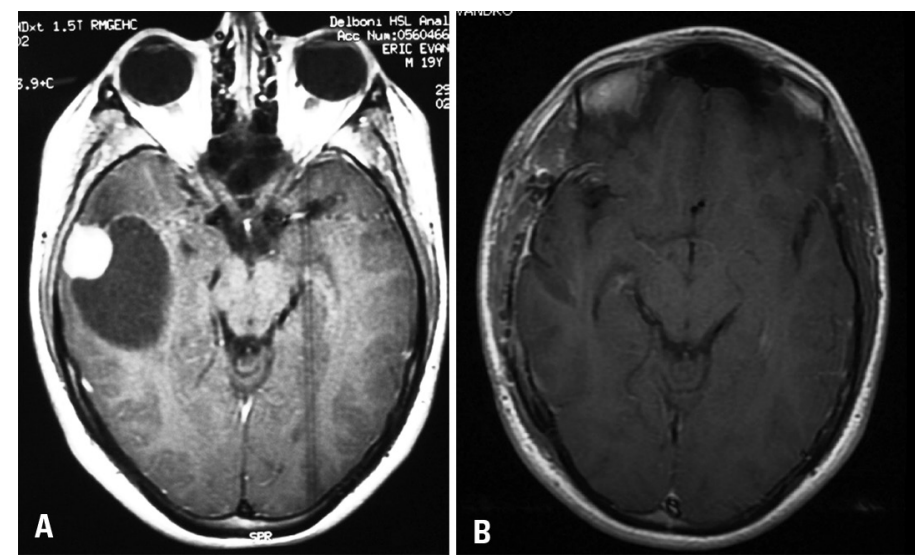
a desmoplastic millieu. With over 50 cases described, the histologic and radiologic spectrum has been well characterized ${ }^{4}$.

Rare tumours with the same morphologic and radiologic features have been described in older subjects ${ }^{5}$. The patients present with an array of symptoms e.g. seizures, weakness and unsteady gait ${ }^{1,5}$. These tumours are generally found in the parietal or temporal lobes, and present as a large cystic mass with peripheral contrast enhancement ${ }^{4}$.

Histopathological examination reveals a well demarcated low grade glial tumour with prominent desmoplasia. Ganglion cells with dysplastic features, clustered focally are also present. Perivascular lymphocytic cuffs and low mitotic activity are also observed ${ }^{5}$.

Immunohistochemically, the glial components are GFAP positive while the ganglion like neuronal cells are positive for NSE, neurofilaments and synaptophysin ${ }^{4,5}$. Like infantile cases, noninfantile desmoplastic gangliogliomas seem to have good prognosis without additional therapy, if a total surgical resection can be performed ${ }^{5}$.
Although accepted as a tumour of infancy, desmoplastic ganglioglioma can also be encountered in older patients. Careful diagnosis and differentiation with other tumours particularly malignant gliomas is important since the therapeutic strategies may differ. In this case, total tumor resection was made and no adjuvant therapy was necessary.

\section{REFERENCES}

1. Qaddoumi I, Ceppa EP, Mansour A, et al. Desmoplastic noninfantile ganglioglioma: report of a case. Pediatr Dev Pathol 2006;9:462-467.

2. VandenBerg SR, May EE, Rubinstein LJ, et al. Desmoplastic supratentorial neuroepithelial tumors of infancy with divergent differentiation potential (desmoplastic infantile gangliogliomas). Report of 11 cases of a distinctive embryonal tumor with favourable prognosis. J Neurosurg 1987;66:58-71.

3. Rout P, Santosh V, Mahadevan A, et al.Desmoplastic infantile gangliogliomaclinicopathological and immunohistochemical study of four cases. Childs Nerv Syst 2002;18:463-467.

4. Fadare O, Mariappan MR, Hileeto D, et al. Desmoplastic infantile ganglioglioma: cytologic findings and differential diagnosis on aspiration material. Cyto Journal 2005;2:1.

5. Onguru O, Celasun B, Gunhan O. Desmoplastic non-infantile ganglioglioma. Neuropathology 2005;25:150-152. 